\title{
Metodología para la organización de los cursos de inglés con fines específicos basada en el problema
}

\author{
Pedro Castro Álvarez \\ Universidad de las Ciencias Informáticas, Cuba \\ pcastro@uci.cu \\ Graciela González Pérez \\ Universidad de las Ciencias Informáticas, Cuba \\ gracielagp@uci.cu \\ Liliana A. Casar Espino \\ Universidad de las Ciencias Informáticas, Cuba \\ lily@uci.cu
}

Resumen: El presente trabajo desarrolla una metodología para la organización de los cursos de inglés con fines específicos basada en el problema como una alternativa a la organización tradicional de la unidad de estudio en torno a un texto escrito. La investigación se realizó en la Universidad de las Ciencias Informáticas de La Habana, Cuba. La metodología propuesta, basada en cinco fases, concede especial importancia al empleo del problema como catalizador de las interacciones y la gestión de información. Al concebir una fase de monitoreo y ayuda, propicia la colaboración entre iguales, en congruencia con el Enfoque Históricocultural de Vigotsky y el Enfoque Comunicativo a la Enseñanza de Idiomas, para de este modo hacer del aprendizaje un proceso significativo.

Palabras claves: Metodología basada en problemas, inglés con fines específicos, Enfoque histórico-cultural, Enfoque comunicativo 
Abstract: This article presents a methodology to organize ESP courses based on problems as an alternative to the traditional organization centered on a written text. The research was carried out at the University of Informatics Sciences in Havana, Cuba. The methodology, based on five phases, gives special importance to the use of the problem as trigger of the interactions and information management. As a phase for monitoring and help is conceived, the methodology encourages collaboration among peers, in keeping with the Historical-cultural Approach and the Communicative Approach to Language Teaching, thus turning learning into a significant process.

Keywords: Problem-based methodology, English for specific purposes, Historical-cultural approach, communicative approach 


\section{Introducción}

En el siglo xxi, la capacidad de asumir responsabilidades laborales demanda más que conocimientos y habilidades básicas. Este reto debe asumirse desde la formación profesional, en la que deben crearse las condiciones y oportunidades para que los estudiantes aprendan también a asumir sus deberes, desarrollar su autoconfianza, resolver problemas, trabajar colaborativamente, comunicar ideas y convertirse en innovadores en sus áreas de desempeño.

La sociedad contemporánea plantea a los especialistas tantos problemas (necesidades sociales) que resulta imposible durante la formación adquirir todos los conocimientos y habilidades que se necesitarán para realizar las actividades inherentes a la práctica profesional.

El Inglés con Fines Específicos (ESP) es la respuesta de la enseñanza de idiomas a las necesidades del empleo del idioma extranjero que surgen en determinados contextos ocupacionales. Tres razones dan lugar al surgimiento del ESP: las demandas provocadas por los cambios a nivel mundial, una revolución lingüística y la tendencia hacia la enseñanza centrada en el estudiante (Gatehouse, 2001): la expansión sin precedentes en la actividad científica, técnica y económica a escala mundial, cuya principal lengua de difusión es el inglés; la revolución en la teoría lingüística encaminada a enfocar las formas en que el idioma se utiliza en la comunicación real; y el desarrollo de la psicología, la cual comenzó a prestar más atención a las formas en que los estudiantes aprenden un idioma.

Un análisis de la manera en que se imparte tradicionalmente el inglés con fines específicos muestra que predomina lo que Bosswood (1990) llama «lección centrada en el texto». En este tipo de lección, ya sea el profesor o el autor del curso preselecciona un material escrito (auténtico, modificado o elaborado especialmente para la actividad) sobre un tema de la disciplina específica. Este texto tiene como finalidad servir de punto de partida para el aprendizaje de aspectos puntuales de la lengua extranjera, como son nuevos vocablos, estructuras gramaticales, modelos de formación de palabras, etc. Asimismo, el texto deviene base para la ejecución de tareas como la extracción de información relevante, la traducción a la lengua materna, el resumen de ideas fundamentales así como la discusión y la presentación de temas afines.

Sin embargo, este enfoque tradicional no logra satisfacer todas las demandas profesionales en la misma medida en que es imposible abarcar en una asignatura, o incluso un conjunto de ellas, la diversidad de contenidos necesarios. Esta situación se hace más evidente a la luz del desarrollo de las tecnologías de la información y las comunicaciones, en particular la Internet. 
La creciente popularidad de la llamada «red de redes» como vía fundamental de compartir información ha tenido un gran impacto en la manera en que se publican y se seleccionan los contenidos. De una parte, es posible publicar de forma más «democrática». Por otra, es necesario ser mucho más crítico ante tal democracia. Si una vez la información publicada en «blanco y negro» garantizaba cierto margen de confiabilidad, hoy en día, el lector o investigador es responsable en primera instancia de evaluar $\mathrm{y}$ «filtrar» las ideas encontradas.

Además, como sugieren Nussbaum y Novick (1982: 186), la enseñanza tradicional, dentro de la que se incluye la lección centrada en el texto, raras veces proporciona a los estudiantes las oportunidades de experimentar conflictos cognitivos.

En cambio, la utilización de un enfoque basado en el problema permite en primer lugar aprender métodos de obtener nuevos conocimientos y desarrollar nuevas habilidades de manera independiente sobre la base de conocimientos y habilidades existentes.

Según Ormond (2006: 2), la resolución de problemas consiste en el empleo de conocimientos y habilidades existentes para responder una pregunta no resuelta o una situación contradictoria. El aprendizaje basado en problemas (ABP) es la adopción de la resolución de problemas en el campo de la instrucción. En este sentido, es un enfoque instructivo en el que los estudiantes adquieren nuevos conocimientos y habilidades mientras trabajan de forma colaborativa en la solución de problemas complejos similares a los que se presentan en el mundo exterior y reflexionan sobre sus experiencias (Ormond, 2006:6). El ABP se basa en el trabajo de investigadores como Vigotsky y Dewey, y se relaciona con las teorías constructivistas del aprendizaje, las que afirman que «el conocimiento de todas las cosas es un proceso mental del individuo que se desarrolla de acuerdo a la interacción con el entorno y que este conocimiento es una construcción del ser humano» (Carretero, 1997: 45). Especial atención dentro de esta corriente merece el llamado «Enfoque Histórico-cultural», el cual considera que los factores sociales son determinantes en el desarrollo personal. De tal modo, según este enfoque, la interacción entre los humanos deviene fuente del desarrollo psíquico, y por consiguiente sirve de sustento teórico básico del trabajo colaborativo (Vigotsky, 1987: 203). Finalmente, la concepción de Vigotsky sobre la unidad entre enseñanza y desarrollo postula la dependencia del desarrollo psíquico del contenido y del carácter de la enseñanza.

Un componente fundamental del ABP es que el contenido se introduce en el contexto de problemas complejos de la vida real. De esta manera, cuando se aplica en el aprendizaje de lenguas extranjeras, ayuda a los estudiantes a establecer un 
puente entre el aprendizaje del idioma y los contenidos particulares de su futura profesión. De manera similar, el ABP aumenta el interés intrínseco en la materia, como señala Bruner (1961: 32), quien concluyó que cuando los estudiantes trabajan en la resolución de problemas que perciben como significativos, muestran un interés en aspectos relativos a esos problemas que va más allá de estudiar con el único fin de aprobar un examen. Hunt (1971:19) trata de explicar este resultado al asumir que cuando las personas se enfrentan con una situación que no comprenden fácilmente, buscan la información necesaria para llenar esos vacíos de conocimiento. Investigaciones más recientes, como las desarrolladas por Morales (2012) y Abercrombie et al (2015: 41), demuestran que el aprendizaje basado en problemas ha constituido una herramienta didáctica muy útil para conseguir un alto nivel de motivación y participación del alumnado.

Otro efecto positivo del ABP es que refuerza sobre una base duradera las estrategias metacognitivas (Norman y Schmidt, 1992: 564). En este sentido, Blumberg y Michael (1991: 7) y Valdez et alii (2010: 120) presentan evidencias de que los estudiantes en un ambiente de aprendizaje basado en la resolución de problemas tienden a desarrollar las habilidades de autogestión del conocimiento. También Shin, Haynes y Johnston (1991) lograron demostrar a través de una serie de pruebas pedagógicas administradas al cabo de los diez años de las actividades de aprendizaje que los estudiantes participantes en actividades de resolución de problemas retuvieron mejor los conocimientos aprendidos en comparación con los que habían participado en entornos de aprendizaje tradicionales.

Existen tres funciones del ABP, según Norman y Schmidt (1992: 560). La primera es la adquisición de conocimiento factual, la segunda es el dominio de los principios o conceptos generales que se pueden transferir a la resolución de problemas afines, y la tercera consiste en la apropiación de ejemplos anteriores que pudieran utilizarse en la resolución de problemas similares en el futuro.

La Universidad de las Ciencias Informáticas, localizada en La Habana, es un centro adscrito al Ministerio de Educación Superior de la República de Cuba. En ella se forman ingenieros informáticos cuya principal misión es contribuir al desarrollo de la informatización en el país. Como parte de su preparación reciben inglés como lengua extranjera, distribuida en dos etapas: Inglés con Fines Generales ( 2 cursos o asignaturas) e Inglés con Fines Académicos y Profesionales (3 cursos). Un estudio preliminar basado en la observación participante y el análisis de los resultados del proceso de enseñanza-aprendizaje, fundamentalmente exámenes, muestra que al culminar la etapa final, algunos estudiantes aún mostraban carencias en cuanto al desarrollo de la competencia comunicativa, no lograban interactuar de manera eficiente con información escrita originalmente en inglés, 
no utilizaban habilidades relacionadas con el pensamiento crítico y no podían transferir las habilidades adquiridas a nuevos contextos. Aunque en esta etapa de Inglés con Fines Específicos, se utilizan ocasionalmente tareas y proyectos, predomina un enfoque basado en el texto escrito como punto de partida para el resto de las actividades de aprendizaje de la lengua extranjera. Las actividades que comúnmente se realizan como parte de la formación en idioma extranjero de los futuros profesionales incluyen la identificación de los actos del habla y la extracción de información de un texto escrito así como la redacción de un párrafo y la presentación oral de un tema previamente estudiado.

Ante esta situación y con vistas a sobrepasar las desventajas de la lección centrada en el texto descritas anteriormente, el presente trabajo tiene como objetivo desarrollar una metodología para un curso de inglés con fines específicos basado en la resolución de problemas de la futura profesión.

En esta investigación, se emplean los métodos inductivo-deductivo, sistémico-estructural e histórico lógico en el nivel teórico. Como métodos de nivel empírico se emplean el análisis documental y la observación participante.

\section{Justificación para el cambio}

La necesidad de adoptar un modelo de lección basada en el problema surge primeramente como parte de la idea de que la lección centrada en el texto responde al modelo obsoleto de «entrada-salida», rechazado por los seguidores del Enfoque Histórico-cultural (Brooks y Donato, 1994: 262; Donato y Lantolf, 1991; Frawley y Lantolf, 1984: 146; Platt y Brooks, 1994: 502). Al respecto, dichos autores argumentan que las nociones de «entrada» $y$ «salida» se basan en un modelo de transferencia de información que no representa exactamente las funciones de la interacción humana. Como las características abiertas de los problemas y sus soluciones estimulan el interés y el debate, el discurso interactivo resultante, según Donato y Lantolf (1991), se convierte en una forma de actividad socialmente situada que sirve no solo para presentar la realidad sino también para crearla y transformarla.

Otras tendencias relacionadas con la lectura de textos, como es la teoría transaccional de la lectura (Rosenblatt, 1994: 91), también rechazan la simple localización de significados en el texto y la extracción de tales significados típicas de los modelos más tradicionales. En su lugar, la teoría transaccional define que los significados se encuentran «enraizados» en la experiencia personal del lector $y$ se someten a la reflexión individual. En este sentido, nuestros esfuerzos por articular y explicar nuestro razonamiento para poder compartir esas experiencias conforman significados. De tal modo, se considera que el significado deja de ser 
propiedad del texto para convertirse en un evento único y personal. Sin embargo, la teoría transaccional también define significados sociales extendidos como propiedades emergentes de las experiencias de múltiples lectores. El aprendizaje basado en la resolución de problemas no entra en contradicción con la teoría transaccional, en tanto la adopción de un problema puede aportar a la lectura motivo y significatividad. Además, si este problema es compartido y resuelto a través del trabajo colaborativo, puede también contribuir a la construcción de significados sociales extendidos.

$\mathrm{Al}$ respecto, el aprendizaje colaborativo de idiomas, elemento fundamental en la metodología propuesta, se explica probablemente mejor a través de la llamada «interpretación del texto como medio» (Wertsch y Bivens, 1992: 41) de la teoría de Vigotsky. Esta interpretación resta importancia al concepto de tutoraprendiz inherente a la modelación, y en su lugar hace énfasis en cómo todos los participantes del aprendizaje se involucran de manera activa en dar forma al aprendizaje. Una vez más los textos dejan de ser meros vínculos para transmitir información para convertirse en «herramientas del pensar» utilizados para generar nuevos significados mediante la colaboración (Warschauer, 1997: 91).

Los enfoques orientados al texto no pueden incorporar completamente el concepto de competencia comunicativa o explotar las potencialidades del enfoque comunicativo. El análisis de los textos como producto cede entonces ante un énfasis en los procesos de construcción e interpretación textual, amparados en los criterios de que las simulaciones, los estudios de casos y las actividades basadas en tareas y proyectos rinden mejores resultados que las actividades orientadas al idioma tradicionales en muchos contextos de la enseñanza de idiomas con fines específicos (Crookall y Saunders, 1989; citados por Boswood, 1990). Boswood (1990) sugiere que esta brecha teórica pudiera sobrepasarse si el concepto de evento comunicativo se utiliza como estructura unificadora de estos dos campos dispares en un enfoque que llama «comunicación con fines específicos». El centro de este enfoque extendido debe ser un nuevo tipo de análisis de las necesidades que tome al evento comunicativo como centro de la atención. Y es precisamente el problema que puede contribuir a la conformación de ese evento comunicativo.

En segundo lugar, la lección centrada en el texto no contribuye significativamente al desarrollo de habilidades consideradas indispensables en el mundo de hoy. Es indudable que la manera en que se lee ha cambiado: por una parte, hay una mayor necesidad de interpretar textos transmitidos por múltiples medios (multimedia) y construidos de forma no lineal (hipertextos); por la otra, la lectura en línea demanda de un alto desarrollo del pensamiento crítico. En su lugar, el empleo del problema como punto de partida implicaría la aplicación de estrate- 
gias de búsqueda en redes y bases de datos, la consulta de múltiples fuentes de información de variada naturaleza, y sobre todo, la capacidad de discernir y tomar decisiones en torno al contenido de los mismos. Al mismo tiempo, de acuerdo con Asbaugh y Kasten (1995: 23), fortalecería habilidades comunicativas, tales como leer y escuchar de forma crítica, leer en busca de detalles, hacer inferencias, sintetizar información y expresar ideas de forma oral y escrita de manera más clara, segura y convincente.

Una tercera razón radica en la naturaleza perentoria de la información hoy en día. La velocidad en que se crean nuevos conocimientos es tal, que el momento de creación de un texto se convierte en un aspecto crítico. De tal modo, los textos preseleccionados podrían perder su vigencia de un curso a otro. Por otra parte, los problemas tienden a ser más persistentes que sus soluciones; lo que significa que un mismo problema, por ejemplo, la seguridad en una red informática, puede perdurar durante años, mientras la comunidad de investigadores y especialistas propone nuevas soluciones continuamente.

Además, existe un elemento de generalización que favorece al problema por encima del texto preseleccionado: mientras un texto puede proporcionar la información necesaria en un momento dado, el análisis y solución de problemas desarrolla en los estudiantes conocimientos y habilidades que son fácilmente transferibles a otras situaciones actuales o futuras (Stivers, 1991).

Otro elemento a favor de la lección basada en el problema es que beneficia la integración curricular con los contenidos propios de la especialidad. La búsqueda de la integración curricular se hace tradicionalmente a través de la selección de textos que responden a determinados contenidos. En cambio, los problemas, como fuente generadora de objetivos, se encuentran en la posición primaria dentro de los componentes del proceso de enseñanza-aprendizaje.

Como se ha discutido, varios elementos justifican el cambio de la lección centrada en el texto a la lección basada en el problema. Sin embargo, un cambio de enfoque implica también un cambio en los métodos utilizados, e incluso en las formas de evaluación.

\section{Diagnóstico de las condiciones en que se realiza el proceso de enseñanza-aprendizaje del Inglés con Fines Académicos y Profesionales en la UCI}

El diagnóstico de las condiciones en que se realiza el proceso de enseñanzaaprendizaje en la UCI muestra que existe la disponibilidad tecnológica (redes y computadoras) necesaria para implementar una estrategia metodológica que 
utilice el problema como elemento central de la clase. De manera similar, los docentes y estudiantes se encuentran capacitados para emplear la tecnología disponible. No obstante, se manifiestan debilidades en los docentes graduados de lengua inglesa para enfrentar problemas tan complejos como los que se pueden presentar en el área de las ciencias informáticas.

El análisis de los documentos curriculares también demuestra la factibilidad del cambio propuesto. La concepción curricular de la carrera adopta un modelo semi-abierto, atendiendo a la descripción de Rita M. Álvarez de Zayas (1997: 21), quien define a este tipo de currículo como «currículo diseñado a nivel central pero en el cual se deja previsto el ajuste, cambio, enriquecimiento de su contenido y estructura». El docente y la institución tienen un margen para realizar cambios en cuanto al número de horas de las asignaturas, los objetivos específicos, etc. Este diseño concede gran importancia a la integración curricular a través de la modelación de sus componentes, y tiende a la interdisciplinaridad, la cual se manifiesta a través de la integración vertical en planes directores y estrategias curriculares, así como horizontal a través de la llamada asignatura integradora. De la misma manera se aprecian objetivos integradores por año. Este modelo de integración curricular reconoce al problema como núcleo integrador de la labor de las diferentes disciplinas.

Figura 1. Relaciones entre los componentes curriculares

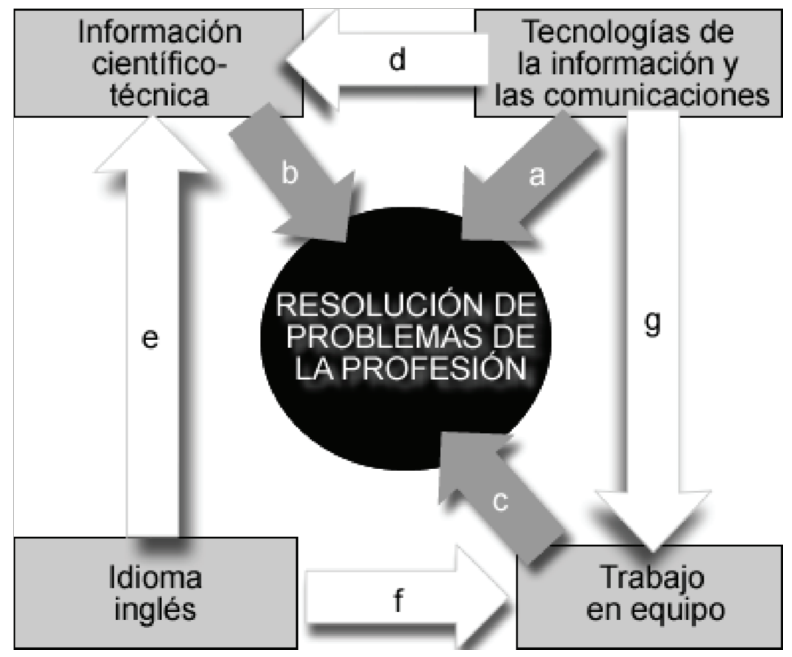

En la Figura 1 se ilustran las relaciones entre los componentes motivos de estudio reflejados en los documentos curriculares. La capacidad del ingeniero informático para resolver problemas relacionados con su profesión constituye el 
núcleo de los estudios. Estos problemas pueden ser solucionados directamente con el concurso de la información científico-técnica (b), que aporta el acceso al conocimiento sobre el tema; las tecnologías informáticas (a), que facilitan el procesamiento de datos; y el trabajo en equipo (c), necesario para solucionar los complejos problemas de la ciencia y la técnica modernas. Por otra parte, el conocimiento de un idioma extranjero contribuye a ampliar el acceso a la información (e) y permite la comunicación con profesionales de otros países para resolver problemas como parte de un equipo. Finalmente, las tecnologías de la información y las comunicaciones hacen un aporte indirecto a la resolución de problemas al potenciar el acceso a la información científico-técnica (d) y el trabajo en equipo (g).

\section{Estrategia metodológica para desarrollar una lección de inglés con fines específicos basada en el problema}

En general, el ABP utiliza un catalizador como contexto para que los estudiantes adquieran conocimientos básicos unidos a habilidades de resolución de problemas (Zhang, 2002; Finkle y Torp, 1995). A diferencia de la clase centrada en el texto, la lección basada en el problema se desarrolla en pequeños grupos de discusión que cuentan con la ayuda gradual de un facilitador, en tanto los estudiantes asumen un mayor grado de responsabilidad como parte fundamental de sus experiencias de aprendizaje (Barrows y Tamblyn, 1980:23; Hmelo-Silver y Barrows, 2006:37; Kwon, Liu y Johnson, 2014). De tal modo, el objetivo fundamental no es promover el conocimiento de los hechos, sino estimular el aprendizaje activo y autónomo, mientras los estudiantes identifican el conocimiento previo sobre el problema, y luego determinan qué necesitan investigar, cuáles preguntas son relevantes en la investigación y qué acciones necesitan emprender (Elizabeth, 2012:53).

Una serie de pasos deben anteceder al diseño del programa del curso propiamente dicho. Estos deben ir encaminados a garantizar un aporte sustantivo a la formación profesional. De igual modo, deben facilitar la integración curricular en forma de interdisciplinariedad:

1. Identificación de los problemas fundamentales de la profesión a partir del análisis de los documentos curriculares.

2. Consulta con los profesores de la especialidad en relación a los problemas identificados en los documentos de la carrera y otros aportes basados en su experiencia profesional. 
3. Organización del programa de las asignaturas correspondientes tomando como referencias los problemas anteriormente identificados.

4. Adopción de un enfoque basado en la tarea para cada unidad de estudio.

5. Diseño del sistema de evaluación contextualizado en el desempeño del profesional.

6. Valoración sistemática de los problemas y los temas seleccionados para comprobar su vigencia.

Aunque es posible hacer una selección y organización en forma de sistema de los aspectos formales de la lengua extranjera (gramática, organización del texto, funciones comunicativas, actos del habla, etc.) que se deben abordar en cada unidad, se recomienda también enseñarlos de forma incidental, ya que es prácticamente imposible anticipar todas las necesidades que pudieran aparecer durante la lectura y análisis de un texto dado. Al respecto, Doghonadze y Gorgiladze (2008) desaconsejan dar respuestas preelaboradas en el proceso de presentación de nuevas estructuras gramaticales y vocabulario, y en su lugar, involucrar a los estudiantes en la formulación y deducción de las mismas a partir de los casos dados.

El proceso de aprendizaje basado en el problema ha sido concebido de varias formas por diferentes autores, quienes proponen su división en varias fases. $\mathrm{Al}$ respecto, Morales y Landa (2004) establecen siete fases: (1) leer y analizar el escenario del problema, (2) realizar una lluvia de ideas, hacer una lista de lo que se conoce, (3) hacer una lista de lo que no se conoce, (4) hacer una lista de lo que necesita hacerse para resolver el problema, (5) definir el problema, (6) obtener información y (7) presentar los resultados.

Por su parte, Exley y Dennik (2009) dividen este proceso en siete fases: (1) aclarar términos y conceptos, (2) definir los problemas, (3) analizar los problemas, (4) hacer una lista sistemática del análisis, (5) formular los objetivos de aprendizaje esperados, (6) desarrollar el aprendizaje independiente centrado en los resultados y (7) sintetizar y presentar nueva información.

Sin embargo, las metodologías mencionadas no explicitan una fase encaminada específicamente a la socialización de los resultados obtenidos y por lo tanto no potencian el trabajo colaborativos entre los participantes.

A partir de la concepción de cinco fases propuesta por Karthikeyan y Baskaran (2009:16), los autores de esta investigación proponen una metodología para conformar cada unidad de estudio en la que se estructura los cursos. De tal modo, estas fases se extenderán durante varias actividades lectivas hasta completar el ciclo. 
Al ser concebida para una universidad tecnológica, específicamente dedicada a la formación de los profesionales de la informática, y que cuenta además con la base material necesaria (ordenadores interconectados), la metodología emplea los recursos de la Web 2.0 para potenciar la socialización en forma de monitoreo, ayuda y co-evaluación. Se considera importante además tener en cuenta que en cada una de estas fases, el profesor y los estudiantes tendrán diferentes funciones. En los Anexos, se presenta una actividad modelo basada en la metodología propuesta. Las fases aparecen a continuación:

1. Introducción al tema.

En esta fase, se explicitan a los estudiantes los objetivos de aprendizaje. Se parte de un breve texto escrito, aunque igualmente se pueden emplear otros medios como presentaciones, vídeos, diagramas, etc. Este material debe dejar claro el tema y los conceptos a tratar. Igualmente debe emplearse para presentar nuevo vocabulario $\mathrm{u}$ aspectos formales de la lengua extranjera que serán imprescindibles para la ejecución de las fases siguientes. Durante esta etapa, que debe ser breve, el papel del profesor es el de presentador.

2. Presentación del problema.

El profesor, en su función como facilitador, presenta el problema en forma de incógnita, conduce a los estudiantes a deducir información relacionada con el mismo y les proporciona fuentes de información básicas. Los estudiantes deberán comprender la incógnita y estar listos para enfrentar la problemática planteada.

3. Organización en grupos e indagación.

En esta etapa el profesor dividirá a los estudiantes en grupos adoptando las estrategias pedagógicas que considere necesarias. De igual modo, proporcionará acceso a variados recursos como libros, Internet, revistas, etc. Debe garantizar la existencia de un ambiente propicio para el aprendizaje. Los estudiantes deberán comunicarse con sus compañeros, explorar los aspectos conocidos y desconocidos, e investigar el problema a profundidad. El profesor ayudará a formular las preguntas investigativas y diseñar estrategias de búsqueda, incluida la identificación de palabras claves.

4. Monitoreo y ayuda

El profesor, actuando como facilitador, controla el progreso de los estudiantes y les brinda el apoyo necesario cuando se presentan dificultades con el idioma extranjero pero debe evitar inmiscuirse en la resolución del problema. Los estudiantes deben realizar una selección crítica de las fuentes, aplicando estrategias de lectura. Deben tomar en cuenta aspectos 
como fuente, autor, fecha, etc. Los estudiantes deben desarrollar discusiones constructivas entre iguales para generar posibles soluciones al problema. En esta fase ocurre la mayor parte de lo que Vigotsky (1978:45) llama «aprendizaje como actividad social» ya que a partir del intercambio de ideas, los estudiantes aportan puntos de vista diferentes ante un mismo problema. El profesor potencia el aprendizaje al moderar la discusión y resaltar los aspectos que requieren mayor atención.

5. Evaluación de los resultados.

El profesor, desde su posición como facilitador, deberá dar a los estudiantes la oportunidad de compartir sus resultados. Los estudiantes socializan sus resultados en forma de presentación o informe. De manera similar, evalúan el desempeño del profesor/facilitador así como su propio desempeño y el de sus compañeros. También examinan las dificultades encontradas, no solo en los aspectos puramente formales de la lengua extranjera, sino también en los contenidos de la disciplina específica y las estrategias para abordar la resolución del problema. Esta evaluación del proceso, unida a la que realiza el profesor, sirve para comprobar el cumplimiento de los objetivos de aprendizaje. De este modo, a partir de las carencias detectadas, el profesor presenta actividades de seguimiento $y$ brinda retroalimentación.

Las fases propuestas se pueden implementar tanto en actividades presenciales como en línea. Sin embargo, los recursos de la red pueden contribuir significativamente en algunas de ellas. Aunque pudieran utilizarse formas relativamente simples como listas de distribución vía correo electrónico o carpetas compartidas, se considera ideal el empleo de un entorno virtual de aprendizaje, de manera que los participantes puedan utilizar las diferentes herramientas disponibles que permiten la publicación de resultados así como la discusión, tanto asíncrona como sincrónica.

Al respecto, los foros de discusión y la mensajería electrónica pueden resultar útiles durante la fase de monitoreo y ayuda. Por su parte, las wikis y las presentaciones virtuales son más ventajosas durante la fase de evaluación de los resultados (Jucevičienè y Valinevičienè, 2010).

Un factor crítico en todo el proceso descrito es la selección del problema. Barbara Duch (1999) señala los atributos esenciales de un «buen problema»: Un problema eficaz debe motivar el interés de los estudiantes por comprender a fondo los conceptos que se presentan. Debe relacionar a la asignatura con el mundo real. Los buenos problemas demandan de los estudiantes la toma de decisiones basadas en hechos, información, lógica y razonamiento. A los estudiantes se les 
debe pedir que justifiquen sus decisiones y su razonamiento basados en los principios aprendidos. Igualmente, demandan que los estudiantes sean capaces de definir qué deducciones son necesarias, qué información es relevante y qué pasos o procedimientos son necesarios para resolverlos. También Gijselaers (1996:12) insiste en la complejidad de las problemáticas para que estas sean significativas a los estudiantes y permitan obtener la solución desde diferentes ángulos.

Es también importante que los objetivos de la asignatura se incorporen a los problemas, conectando el conocimiento anterior con los nuevos conceptos, y vinculando el nuevo conocimiento a conceptos de otras disciplinas. Para ello, cada unidad deberá trazarse objetivos de aprendizaje, no solamente para el área de especialización sino también para el aprendizaje de la lengua extranjera, los cuales expresarán qué se espera que los estudiantes aprendan o sean capaces de hacer.

Otro aspecto de relevancia es que el problema demande necesariamente la cooperación de todos los miembros del grupo para su solución. Para esto, las preguntas iniciales deben ser abiertas (no limitadas a una respuesta correcta única), relacionarse con conocimientos ya adquiridos, mostrar aspectos controvertidos que provoquen respuestas disímiles; de este modo, lograrán atraer a la mayoría de los estudiantes a la discusión. Esta estrategia está destinada a hacer que los estudiantes trabajen en grupo, propiciando una colaboración auténtica.

En este sentido, el trabajo en grupo y la exposición que ocurren fundamentalmente durante la fase de monitoreo y ayuda así como en la de evaluación de los resultados generan conflictos cognitivos en los estudiantes, al enfrentar variadas alternativas, que surgen no solo de la búsqueda de información sino también de la propia labor creativa de los participantes (Glaser, 1991). Estos conflictos devienen indispensables para alcanzar aprendizajes significativos, ya que ayudan a establecen relaciones sólidas entre lo ya sabido y lo nuevo aprendido. De tal modo, los estudiantes revisan, modifican y enriqueces sus estructuras de pensamiento (Coll, 2002).

Como se ha descrito anteriormente, y en concordancia con las ideas de Stepien y Gallagher (1993), durante todo el ciclo, pero fundamentalmente en la fase de monitoreo y ayuda, los profesores deben actuar la mayor parte del tiempo como facilitadores del aprendizaje, sirviendo de modelo, pensando en voz alta y practicando los comportamientos que a su vez esperan de los estudiantes.

La metodología propuesta, en la que se concibe la enseñanza-aprendizaje de la lengua extranjera mediante la ejecución de una serie de tareas basadas en problemas, está en congruencia con el enfoque al ESP llamado «comunicación con fines específicos» (Boswood, 1990) porque en la misma, no se concibe al texto preseleccionado por el profesor como punto de partida del aprendizaje de idio- 
mas, sino al propio evento comunicativo que surge de la tarea planteada. Esto permite integrar la formación profesional con el desarrollo lingüístico al situar el aprendizaje en el contexto significativo de los procesos académicos y ocupacionales y construir relaciones entre ambos. Incluye actividades que propician el desarrollo de estrategias cognitivas como explorar, buscar y seleccionar información; estrategias socio-afectivas como colaborar entre iguales, interactuar con alumnos y especialistas, y solicitar o suministrar ayuda; y estrategias metacognitivas como reflexionar sobre la ejecución de las tareas, monitorear su comprensión y evaluar su propio progreso.

Esta metodología permite al Inglés con Fines Específicos avenirse a los postulados de la enseñanza comunicativa de idiomas. Al respecto, concibe al idioma extranjero como una herramienta para solucionar problemas que demandan el empleo de la comunicación y se centra en propiciar al estudiante oportunidades concretas y significativas de utilizar el idioma aprendido.

Concuerda además con las ideas expuestas por Doghonadze y Gorgiladze (2008), para quienes el principal objetivo desde el punto de vista práctico del enfoque comunicativo en la enseñanza de idiomas es que los estudiantes dominen no solo cierta cantidad de contenidos lingüísticos sino también que sean capaces de utilizar dichos contenidos en las situaciones nuevas que surgen continuamente.

La justificación fundamental del empleo del aprendizaje colaborativo es que aproxima las tareas al «mundo real» en comparación con las estrategias didácticas tradicionales. Las actividades que demandan la cooperación entre los individuos reflejan la forma en que se ejecutan las tareas en la práctica (Vigotsky, 1978: 215; Bridges et alii, 2015: 122), en la que el lenguaje desempeña un papel clave. Por otra parte, prepara a los estudiantes para trabajar en equipos, lo que resulta indispensable si esperamos de ellos que puedan resolver complejos problemas interdisciplinarios como parte de su futura vida laboral.

Desafortunadamente, la aplicación de un enfoque basado en el problema, como se ha mencionado anteriormente, demanda del profesor de idiomas un nivel adecuado de conocimientos relacionados con la especialidad, para lo cual puede no estar preparado (Lopatina et alii, 2015: 137). Además, tiene la desventaja de ser un proceso que exige mucho tiempo tanto del profesor, durante la selección y planeamiento de la actividad, como del estudiante para poder llegar a una solución. Lleva más tiempo llegar a conclusiones de manera totalmente independiente o bajo la tutela del profesor que a través de una explicación tradicional. Es un enfoque que requiere grandes esfuerzos intelectuales de ambas partes también. Pero, al decir de Doghonadze y Gorgiladze (2008), es un enfoque indispensable si queremos que nuestros estudiantes dominen no solo los conocimientos esen- 
ciales, los cuales pierden vigencia con mucha rapidez, sino también las habilidades para gestionar continuamente su propio conocimiento.

\section{Conclusiones}

La adopción de un enfoque basado en la resolución de problemas de la profesión constituye una necesidad y una posibilidad en el contexto de la enseñanza de inglés con fines académicos y profesionales en la Universidad de las Ciencias Informáticas de La Habana, Cuba, debido a que el enfoque basado en el texto utilizado tradicionalmente no logra cumplir las expectativas en la formación de un ingeniero de las ciencias informáticas capaz de gestionar la información en inglés que necesita para dar solución a los múltiples problemas propios de su desempeño profesional. Por otra parte, el estudio de los documentos de la carrera demuestra que el problema constituye el núcleo de la organización curricular. Asimismo, la Universidad cuenta con los recursos humanos y tecnológicos que le permiten utilizar la metodología propuesta.

La metodología propuesta, basada en cinco fases, concede especial importancia al empleo del problema como catalizador de las interacciones y la gestión de información. Al concebir una fase de monitoreo y ayuda, propicia la colaboración entre iguales, en congruencia con el Enfoque Histórico-cultural de Vigotsky y el Enfoque Comunicativo a la Enseñanza de Idiomas, para de este modo hacer del aprendizaje un proceso significativo.

\section{Referencias bibliográficas}

Abercrombie, S; J. Parkes y T. McCarty (2015). «Motivational Influences of Using Peer Evaluation in Problem-Based Learning in Medical Education». Interdisciplinary Journal of Problem-Based Learning, 9(1), 33-43. [<http:// dx.doi.org/10.7771/1541-5015.1501> ].

Álvarez de Zayas, R. M. (1997). Hacia un curriculum integral y contextualizado. La Habana: Editorial Academia.

Asbaugh, C. y Kasten, K. (1995). Educational leadership: case studies for reflective practice. New York: Longman.

Barrows, H. S. y Tamblyn, R. M. (1980). Problem-based Learning - An Approach to Medical Education. New York: Springer.

Blumberg, P. y Michael, J. A. (1991). «The Development of Self-directed Learning Behaviors in a Partially Teacher-centered, Problem-based Learning Curriculum». Teach. Learn. Med. 4:3-8. 
Boswood, T. (1990). «Communication for Specific Purposes: Establishing the Communicative Event as the Focus of Attention». En: Actas de 4th Annual International Conference on Pragmatics and Language Learning (celebrada el 4 de abril de 1990). Urbana-Champaign: University of Illinois.

Bridges, S. M.; Corbet, E. F. y Chan, L. K. (2015). «Designing problem-based curricula: The role of concept mapping in scaffolding learning for the health sciences». Knowledge Management E E-Learning, 7(1), 119-133.

Brooks, F. y Donato, R. (1994). «Vygotskyan Approaches to Understanding Foreign Language Learner Discourse during Communicative Tasks». Hispania, 77, 262-264.

Bruner, J. S. (1961). «The act of discovery». Harvard Educational Review 31: $21-32$.

Carretero, M. (1997). ¿Qué es el constructivismo. Constructivismo y educación, Desarrollo cognitivo y aprendizaje. México: Editorial Progreso, 39-71.

Coll, C. (2002). «Concepciones y tendencias actuales en psicología de la educación». En: Coll, C.; J. Palacios, A. Marchesi (comp.) Desarrollo psicológico y educación. Vol 2. Psicología de la Educación Escolar. Madrid: Alianza Editorial.

Dognonadze, N. y Gorgiladze, G. (2008). «Problem Solving in Teaching Foreign Languages to Students». IBSU Scientific Journal, 2(1).

Donato R. y Lantolf, J. P. (1991). «Communication and social interaction: Conduit or Cognitive Activity». En: Actas del Biennial Meeting of the Society for Research in Child Development. Seattle, WA.

Duch, B. (1999). Problems: A Key Factor in PBL. Delaware: University of Delaware. [<http://www.udel.edu/pbl/curric/chem103-prob.html >].

Elizabeth, M. A. (2012). «Problem-Based Learningः A Source of Learning Opportunities in Undergraduate English for Specific Purposes». The International Journal of Social Sciences, 3(1), 47-56.

Exley, K. y Dennick, R. (2009). Giving a lecture: from presenting to teaching. London: Routledge.

Finkle, S. y Torp, L. (1995). Introductory Documents. Chicago: Illinois Math and Science Academy.

Frawley, W. y Lantolf, J. P. (1984). «Speaking and Self-order: A Critique of Orthodox L2 Research». Studies in Second Language Acquisition, 6(2), $143-$ 159.

Gatehouse, K. (2001). «Key Issues in English for Specific Purposes (ESP) Curriculum Development». The Internet TESL Journal, 7(10). [<http:// www.cybertrails.com/ jhoey/> ] 
Giselaers, W. H. (1996) Bringing Problem Based to Higher Education. Theory and Practice. San Francisco: Jossey-Bass Publisher.

Glaser, R. (1991). "The Maturing of the relationship between the science of learning and cognition and educational practice». Learning and Instruction $1(1), 129-144$.

Hmelo-Silver, C. E. y Barrows, H. S. (2006). «Goals and Strategies of a Problem-based Learning Facilitator». The Interdisciplinary Journal of Problembased Learning, 1(1), 21-39.

Hunt, J. McV+ (1971). «Intrinsic Motivation: Information and Circumstances»+ En: Personality Theory and Information Processing, H. M. Schroeder y P. Suedfeld, (eds.) 12-23, New York: Ronald.

Jucevičiené, P+ y Valinevičiené, G. (2010). «A Conceptual Model of Social Networking in Higher Education». Electronics and Electrical Engineering 6(102), 55-58.

Karthikeyan, R. y Baskaran, G. (2009). «Using Problem Based Learning Technique in Teaching English Grammar». Language in India. Strength for Today and Bright Hope for Tomorrow. 9(10), 12-18.

Kwon, K.; Y. Liu y Johnson, L. P. (2014). "Group regulation and socialemotional interactions observed in computer supported collaborative learning: Comparison between good vs, poor collaborators». Computers $\mathcal{E}$ Education 78, 185-200 [<http://www.elsevier.com/locate/compedu > ].

Morales, F. M. (2012). «Uso del aprendizaje basado en problemas como metodología activa». En: Actas del $1^{\circ}$ Congreso Internacional de Educación Media y Superior. México: Centro de Estudios e Investigaciones para el Desarrollo Docente.

Morales, P. y Landa, V. (2004). «Aprendizaje basado en problemas». Theoria, 13(1), 145-157.

Norman, G., y Schmidt, H. (1992). "The Psychological Basis of ProblemBased Learning: A Review of the Evidence». Academic Medicine, 67(9), 557565.

Nussbaum, J, y Novick, S. (1982). «Alternative Frameworks, Conceptual Conflict and Accommodation: Toward a Principled Teaching Strategy». Instructional Science, 11, 183-200.

Ormond, J. E. (2006). Essentials of Educational Psychology. New York: Pearson Merrill Prentice Hall.

Platt, E., y Brooks, F+ (1994). «The «Acquisition-rich» Environment Revisited.» Modern Language Journal, 78, 497-511. 
Rosenblatt, L. M. (1994). "The transactional model of reading and writing». En: R. B. Ruddell, M. R. Ruddell y H. Singer (Eds.), Theoretical models and processes of reading. Newark, DE: IRA.

Sandoval, V;; Blanco, S.; Astorga, B. y Manosalva, S. (2015). Teorías constructivistas del aprendizaje (Tesis para optar por el título de Profesora en Educación Diferencial), Santiago de Chile: Universidad Academia de Humanismo Cristiano.

Shin, J. H.; Haynes, R. B. y Johnston, M. (1991). «The Effect of Problembased Self-directed Undergraduate Education on Lifelong Learning». En: Actas de Canadian Society for Clinical Investigation meeting. (celebrado en septiembre de 1991). Montreal, Canadá.

Stepein, W.y Gallagher, S. (1993). «Problem-Based Learning: as Authentic as It Gets». Educational Leadership, 50 (7). [<http://www.ascd.org/ readingroom/edlead/9304/stepien.html> ].

Stivers, J. (1991). «An Introduction to Case Use in Teacher Education». En: Actas de Annual Meeting of the Confederated Organizations for Teacher Education. New York: American Association of Colleges for Teacher Education and The New York State Association of Teacher Educators.

Valdez, M. T.; Agreira, C. F. y Ferreira, C. M. (2010). «Teaching, Learning and Exploring the Use of Project-Based Learning». Education in Electronics and Electrical Engineering 9(105), 117-120.

Vigotsky, L. S. (1989). Obras completas (Vol. 5). La Habana: Pueblo y Educación.

- (1987): Historia del desarrollo de las Funciones Psíquicas Superiores. La Habana: Editorial Científico Técnica.

Lopatina, O. V. et alii (2015). «Role of Foreign Language Teacher Shaping Students' Research Skills». Asian Social Science 11 (4), 135-140. [<http // dx.doi.org/10.5539/ass.v11n4p135> ].

Warschauer, M. (1997). «A Sociocultural Approach to Literacy and its Significance for CALL». En: K. Murphy-Judy y R. Sanders (Eds.), Nexus: The convergence of research $\mathcal{E}$ teaching through new information technologies (pp. 88-97). Durham: University of North Carolina.

Wertsch, J. y Bivens, J. A. (1992). «The social origins of individual mental functioning: Alternatives and perspectives». The Quarterly Newsletter of the Laboratory of Comparative Human Cognition, 14(2), 35-44.

Zhang, G. (2002). Using Problem-based Learning and Cooperative Group Learning. [<http://science.uniserve.edu.au/pubs/china/vol 1/guiling.pdf $>$ ]. 


\section{Anexos}

\section{Actividad de ejemplo}

\begin{tabular}{|c|c|}
\hline Título: & Safer connections \\
\hline $\begin{array}{l}\text { Ubicación } \\
\text { en el currículo: }\end{array}$ & $\begin{array}{l}\text { Asignatura Idioma Extranjero V, perteneciente a la etapa de Inglés } \\
\text { con fines profesionales. Unidad } 1 \text {. }\end{array}$ \\
\hline Objetivos: & $\begin{array}{l}\text { Los estudiantes: } \\
\text { - } \quad \text { darán respuesta a problemas relacionados con la seguridad en } \\
\text { redes informáticas, } \\
\text { - } \quad \text { formularán preguntas investigativas y palabras claves acordes } \\
\text { al tema investigado, } \\
\text { - evaluarán los contenidos de diversas fuentes de información } \\
\text { en línea en inglés, } \\
\text { - } \quad \text { utilizarán el inglés para compartir y discutir la información } \\
\text { encontrada así como las soluciones propuestas al problema } \\
\text { planteado, tanto de forma oral como escrita. } \\
\text { redactarán informes en idioma inglés con los resultados } \\
\text { encontrados. }\end{array}$ \\
\hline $\begin{array}{l}\text { Preguntas de } \\
\text { seguimiento: }\end{array}$ & $\begin{array}{l}\text { Las siguientes preguntas servirán para dar seguimiento a los diferentes } \\
\text { aspectos del proceso de resolución del problema propuesto: } \\
\text { ¿Determina el estudiante la naturaleza del problema y posibles } \\
\text { interrogantes? ¿Determina el estudiante qué información adicional } \\
\text { necesita para solucionar el problema planteado? ¿Estudia las } \\
\text { diferentes respuestas preliminares al problema planteado y revisa las } \\
\text { interrogantes? ¿Utiliza el índice y del motor de búsqueda. ¿Organiza } \\
\text { el estudiante la información adquirida? ¿Utiliza más de una fuente } \\
\text { de información? ¿Evalúa el estudiante la calidad de la información } \\
\text { en las diferentes fuentes? ¿Re-expresa el estudiante los resultados } \\
\text { de su investigación con sus propias palabras? ¿Distingue hechos } \\
\text { de opiniones? ¿Evalúa adecuadamente la calidad de la información } \\
\text { recopilada? ¿La información adquirida refleja las necesidades de la } \\
\text { tarea? ¿La solución se expresa de manera clara, concisa y apropiada? } \\
¿ E l \text { estudiante referencia los recursos utilizados? ¿Qué aspectos del } \\
\text { idioma extranjera necesitan retroalimentación y práctica adicional? }\end{array}$ \\
\hline
\end{tabular}




\begin{tabular}{|c|c|}
\hline $\begin{array}{l}\text { 1ra fase } \\
\text { Presentación: }\end{array}$ & $\begin{array}{l}\text { La actividad lectiva inicia a partir de un warm-up, en este punto } \\
\text { el profesor fomenta la discusión en torno al tema de los virus de } \\
\text { ordenadores. } \\
\text { A continuación presenta un breve texto inicial tomado de PC } \\
\text { Magazine: } \\
\text { You can set up a network firewall. You can run software that fights vi- } \\
\text { ruses, spyware, and spam. You can install patches that shore up vulner- } \\
\text { abilities in your operating system. But no matter how diligent you are, } \\
\text { the number-one, nastiest, most frustrating problem facing computer users } \\
\text { today-whether they're aware of it or not-is that machines are far too } \\
\text { vulnerable. There are too many exploitable holes in the software we run, } \\
\text { and today's security methods aren't always nimble enough to keep up } \\
\text { with ever-evolving methods of attack. } \\
\text { Even if you've been lucky enough to evade malicious viruses and spyware, } \\
\text { you're still receiving bucket loads of spam, which can also be malicious- } \\
\text { thanks to such online confidence tricks as phishing and pharming. This } \\
\text { unending barrage of nefarious software slows our PCs and keeps them } \\
\text { from behaving like they're supposed to. At its worst, it can compromise } \\
\text { our privacy-and empty our wallets. } \\
\text { En este momento, el profesor presenta los objetivos de la unidad lec- } \\
\text { tiva. A continuación verifica la comprensión del vocabulario funda- } \\
\text { mental relacionado con el tema. }\end{array}$ \\
\hline $\begin{array}{l}\text { 2da fase } \\
\text { Presentación } \\
\text { del problema: }\end{array}$ & $\begin{array}{l}\text { Durante esta fase, el profesor presenta a los estudiantes la siguiente } \\
\text { incógnita: } \\
\text { How can we deal with the risks of security in our computers } \\
\text { and networks? } \\
\text { A continuación orienta la indagación en la Web: } \\
\text { Examine multiple alternatives. You can start your search at } \\
<h t t p: / / w w w . p c m a g . c o m />\text {, }<h t t p: / / w w w . p c w o r l d . c o m>\text {, } \\
<h t t p: / / w w w . w i r e d . c o m>\text { and }<h t t p: / / w w w . p c a d v i s o r . \\
\text { co.uk }>\text {. Then, use the links provided in these general-public } \\
\text { magazines to move on to sources that are more specialized. } \\
\text { Un breve diálogo permitirá corroborar si los estudiantes } \\
\text { han comprendido las orientaciones provistas. }\end{array}$ \\
\hline
\end{tabular}




\begin{tabular}{|c|c|}
\hline $\begin{array}{l}\text { 3ra fase } \\
\text { Organización } \\
\text { en grupos e } \\
\text { indagación: }\end{array}$ & $\begin{array}{l}\text { El profesor organiza los equipos de trabajo procurando incluir en cada } \\
\text { uno de ellos estudiantes con diferentes grados de desarrollo de las } \\
\text { habilidades. Los equipos trabajan de forma presencial examinando el } \\
\text { texto inicial y los resultados de la búsqueda de información preliminar } \\
\text { y contrastándolos con los conocimientos que ya poseen sobre el tema. } \\
\text { Mientras los estudiantes colaboran el profesor monitorea su labor, } \\
\text { cuidando de que efectivamente utilicen la lengua extranjera para } \\
\text { la comunicación. En este caso, el docente ayuda a los estudiantes } \\
\text { a examinar el problema en sus múltiples aristas. El profesor debe } \\
\text { prever algunas de las preguntas investigativas que pudieran surgir, } \\
\text { como las siguientes: } \\
\text { - What security methods are used nowadays? } \\
\text { - How can we prevent software backdoors? } \\
\text { - How can we face virus attacks effectively? } \\
\text { - How can we avoid being victims of spamming? } \\
\text { - How can we protect our privacy? } \\
\text { Finalmente, los estudiantes deben formular estrategias de búsqueda. } \\
\text { En este momento deben identificar palabras claves, tales como } \\
\text { antivirus, backdoors, computer viruses, phishing, pharming, spams, } \\
\text { network security y privacy. }\end{array}$ \\
\hline $\begin{array}{l}\text { 4ta fase } \\
\text { Monitoreo } \\
\text { y ayuda: }\end{array}$ & $\begin{array}{l}\text { Esta fase se desarrolla de forma no presencial, los estudiantes exploran } \\
\text { recursos de la Internet y hacen una selección crítica de las fuentes. } \\
\text { El profesor distribuye materiales que ayudan al estudiante a tomar } \\
\text { decisiones durante la selección de las fuentes. Se crea un foro de } \\
\text { discusión sobre el tema en el entorno virtual de aprendizaje (Moodle) } \\
\text { en el que los participantes intercambian información y expresan sus } \\
\text { dudas y dificultades. Cada equipo utiliza el recurso Wiki para crear } \\
\text { un texto colaborativo en el que exponen las soluciones propuestas. } \\
\text { El profesor participa en las discusiones que se generan en el foro. } \\
\text { De manera similar, debe llevar un registro de las dificultades que van } \\
\text { apareciendo. }\end{array}$ \\
\hline $\begin{array}{l}\text { 5ta fase } \\
\text { Evaluación de } \\
\text { los resultados: }\end{array}$ & $\begin{array}{l}\text { Se concibe como una actividad presencial, en este punto cada equipo } \\
\text { habrá creado una presentación electrónica, que ha sido publicada } \\
\text { en el entorno virtual de aprendizaje. Los equipos presentan sus } \\
\text { resultados de forma oral ante el resto de sus compañeros. El profesor } \\
\text { asigna roles de oponentes a determinados estudiantes, quienes } \\
\text { formularán preguntas adicionales o criticarán los puntos débiles de } \\
\text { la presentación. Los estudiantes discuten a continuación hasta qué } \\
\text { punto se cumplieron los objetivos previstos. El profesor indaga sobre } \\
\text { las dificultades encontradas. A continuación, basado en las preguntas } \\
\text { de seguimiento, el docente hace énfasis en las carencias detectadas } \\
\text { durante el proceso y brinda retroalimentación. Finalmente, propone } \\
\text { actividades adicionales orientadas a eliminar dichas dificultades. }\end{array}$ \\
\hline
\end{tabular}

